\title{
VARIACIONES ESTACIONALES EN LA GENERACIÓN DE ESCORRENTÍA Y EMISIÓN DE SEDIMENTOS EN UNA LADERA DE LOS MONTES DE MÁLAGA (PROVINCIA DE MÁLAGA)
}

\author{
Juan Francisco Martínez-Murillo \\ José Damián Ruiz Sinoga
}

\begin{abstract}
RESUMEN
En una ladera representativa de los Montes de Málaga, de antiguo manejo agrícola y actualmente cubierta por vegetación de matorral, se han medido las variaciones estacionales de la generación de escorrentía y la emisión de sedimentos en los distintos ambientes que conforman su sistema eco-geomorfológico. Ello fue llevado a cabo mediante dos técnicas: 1) simulaciones de lluvia realizadas en octubre de 2004 (inicio de la estación hidrodinámica activa) y marzo 2005 (mediados de la estación hidrodinámica activa), y 2) seguimiento de la afección de eventos de lluvia reales en parcelas cerradas de diferentes longitudes. Los resultados ofrecen unas variaciones estacionales considerables: i) predominio de las condiciones favorables para la generación de escorrentía en otoño, por la presencia de un suelo extremadamente seco tras el verano, mostrando síntomas de hidrofobia; y ii) predominio de la infiltración a mediados de la estación húmeda una vez que el suelo fue humedeciéndose a lo largo de ésta.
\end{abstract}

\section{ABSTRACT}

The seasonal changes of the runoff generation and sediment emission have been measured at a slope from Montes de Málaga. This slope was cultivated until fifty years ago and it is occupied by shrub vegetation now. Measures were carried out in every one of the environments which composed the eco-geomorphological system of the slope. Two methodologies have been used for the purpose: 1) rainfall simulations during October 2004 (beginning of active hydrodynamic season) and March 2005 (middle of active hydrodynamic season), and 2) closedplots with different longitude. Results shows considerable seasonal changes: i) favourable conditions for runoff generation in Autumn a cause of a soil very dry after the summer showing hydrophoby, and ii) conditions for infiltration at the middle of the wet season once the soil was moistured along the active hydrodynamic season.

(C) Baetica. Estudios de Arte, Geografía e Historia, 28, 2006, 435-465. ISSN: 0212-5099

Facultad de Filosofía y Letras, Universidad de Málaga. Campus de Teatinos, E-29071 Málaga (España) 


\section{INTRODUCCIÓN}

El sistema eco-geomorfológico se define como aquél integrado por el clima, el suelo, la vegetación, el agua y la erosión (Lavee et al. 1998), elementos que interactúan en un modo complejo y variado. El suelo es uno de los elementos del sistema que controla en gran medida su comportamiento hidrológico en el proceso de infiltración del agua (Cantón et al. 2004). Diferentes propiedades del suelo se han definido como factores claves de la hidrodinámica del sistema eco-geomorfológico: contenido de arcillas, costras, contenido de fragmentos rocosos, rugosidad superficial, etc. A pequeña escala, estas propiedades pueden influir en la hidrología y erosión del suelo más que el clima (Cerdà 1998a). Pero uno de los factores más importantes en esta respuesta hidrológica es el contenido de agua del suelo previo a la precipitación (El-Kadi 1987; Beven 2002) que depende del régimen de precipitaciones (Martínez-Fernández 1996; Boix-Fayos 1999; Ceballos et al. 2002; Ramos y Mulligan 2005).

Los suelos tienen su sistema poroso al menos parcialmente llenos de agua a lo largo de la estación húmeda y comienzan a secarse desde la última precipitación antes del verano. Desde esta relación entre precipitación y humedad del suelo, apuntamos dos conceptos más precisos que los de estación seca y húmeda: estaciones hidrodinámicas activas y no activas. La primera comenzaría desde el inicio de la primera precipitación después del largo y seco verano mediterráneo. A lo largo de esta estación activa, el agua está en movimiento sobre y dentro del suelo (escurriendo sobre la superficie del suelo o dentro del suelo, infiltrándose, percolando hacia las aguas subterráneas, evaporándose, etc.). Y la segunda estación se iniciaría desde el momento en el que la humedad del suelo se sitúa de forma constante por debajo del punto de marchitez, y se prolongaría hasta el inicio de la siguiente estación hidrodinámica activa (Martínez-Murillo, 2006).

Procesos hidrofóbicos pueden ser observados al final de la estación hidrodinámica no activa dados los bajos niveles de humedad del mismo. (De Bano 1971; Witter et al. 1991; Ritsema y Dekker 1994; Doerr y Thomas 2000; Doerr et al. 2000). La hidrofobia reduce la capacidad de infiltración del suelo y por tanto incrementa la escorrentía superficial y la erosión del suelo (Imeson et al. 1992; Scott y Schulze 1992; Scott 1993; Shakesby et al. 1993). También puede determinar el movimiento del agua dentro del suelo (Scott y Van Wyk 1990; Lavabre et al. 1993; Ritsema et al. 1993) evolucionando desde el comienzo de las lluvias otoñales hasta que la humedad del suelo no alcanza un cierto equilibrio con su estructura. La sucesión de eventos lluviosos provocan en el suelo la reducción de los procesos hidrofóbicos una vez se ha superado un cierto umbral crítico de humedad, (Dekker y Ritsema 1994; Soto 
et al. 1994) lo que manifiesta que no es una propiedad estática sino que sigue un patrón dinámico en el corto plazo (Doerr et al. 2000; Ferreira et al. 2000; McHale et al. en prensa). En consecuencia, los mecanismos de generación de escorrentía pueden verse modificados a escala de ladera.

Ferreira et al. (2000) observaron mecanismos hortonianos en la estación mediterránea seca cuando el suelo es más hidrofóbico, y una vez superado esta situación, los mecanismos de saturación fueron los predominantes. Aunque la hidrofobia no se identificó en otras investigaciones como el principal factor de la generación de escorrentía, la modificación de los mecanismos de los flujos superficiales a lo largo del año han sido también identificados: escorrentía hortoniana o por exceso de infiltración en verano o con unos bajos contenidos de humedad previos (Yair y Klein 1973; Yair y Lavee 1974, 1976 y 1985; Cerdá i Bolinches 1995; Boix-Fayos 1999; Calvo et al. 2003a; Castillo et al. 2003) y escorrentía por saturación en invierno o ante unos elevados contenidos de humedad previos (Boix-Fayos 1999; Calvo et al. 2003a; Martínez-Murillo 2006). La variabilidad dentro del sistema le dota de mayor complejidad puesto que ambos mecanismos pueden coexistir en el tiempo en una misma ladera debido a que estas pueden ser consideradas mosaicos constituidos por áreas infiltradoras y áreas generadoras (Yair y Klein 1973; Yair y Lavee 1974, 1976, 1985; Yair y Enzel 1987; Cerdá et al. 1995; Nicolau et al. 1996; Puigdefábregas y Sánchez 1996b; Bergkamp 1998a; Cerdà 1997, 1998b; Lavee et al. 1998; Wainwright et al. 2002; Calvo et al. 2003a; Ruiz-Sinoga et al. 2004; Bracken y Kirkby 2005). Así pues, a lo largo de estas laderas pueden definirse zonas desconectadas hidrológicamente, debido a la disposición de la vegetación en las mismas (matorral, suelo desnudo con diferentes tipos de rasgos superficiales, vegetación herbácea, etc.). Cammeraat (2002) establece que la conexión entre áreas infiltradoras y generadoras está dominada por la magnitud, frecuencia y duración de las precipitaciones y controlada por umbrales físicos y biológicos como la humedad previa del suelo, el patrón de la vegetación y la actividad de los micro-organismos. Como distintos autores han apuntado, la consecuencia de esta situación es la presencia de mecanismos de generación de escorrentía de elevada complejidad y variabilidad (Bergkamp 1998b; Lavee et al. 1998; Puigdefábregas 1998; Puigdefábregas et al. 1998, 1999; Boix-Fayos 1999; Calvo et al. 2003a; Güntnert y Bronstert 2004).

Los objetivos de esta investigación han sido los siguientes. En primer lugar, establecer los mecanismos de generación de escorrentía de los diferentes ambientes que componen el sistema eco-geomorfológico de una ladera, localizada en un entorno en transición entre el régimen semiárido y sub-húmedo. En segundo lugar, definir aquellos mecanismos de escorrentía en dos momentos muy importantes desde el punto de vista hidrológico y erosivo debido al riesgo de eventos de lluvias extremos: uno, justo al final de la estación hidro- 
dinámica no activa ante unas condiciones de suelo extremadamente secas, y otra, a mediados de la estación hidrodinámica activa, con un contenido mayor de humedad en el suelo. Es decir, definir el comportamiento hidrológico superficial del suelo, en relación a su contenido de humedad. Finalmente, atendemos también a la evolución de la escorrentía y el transporte de sedimentos desde el comienzo de la estación hidrodinámica activa y su correspondencia con procesos de conectividad hidrológica a lo largo de la ladera.

\section{FIGURA 1 \\ LOCALIZACIÓN DEL ÁREA DE ESTUDIO}

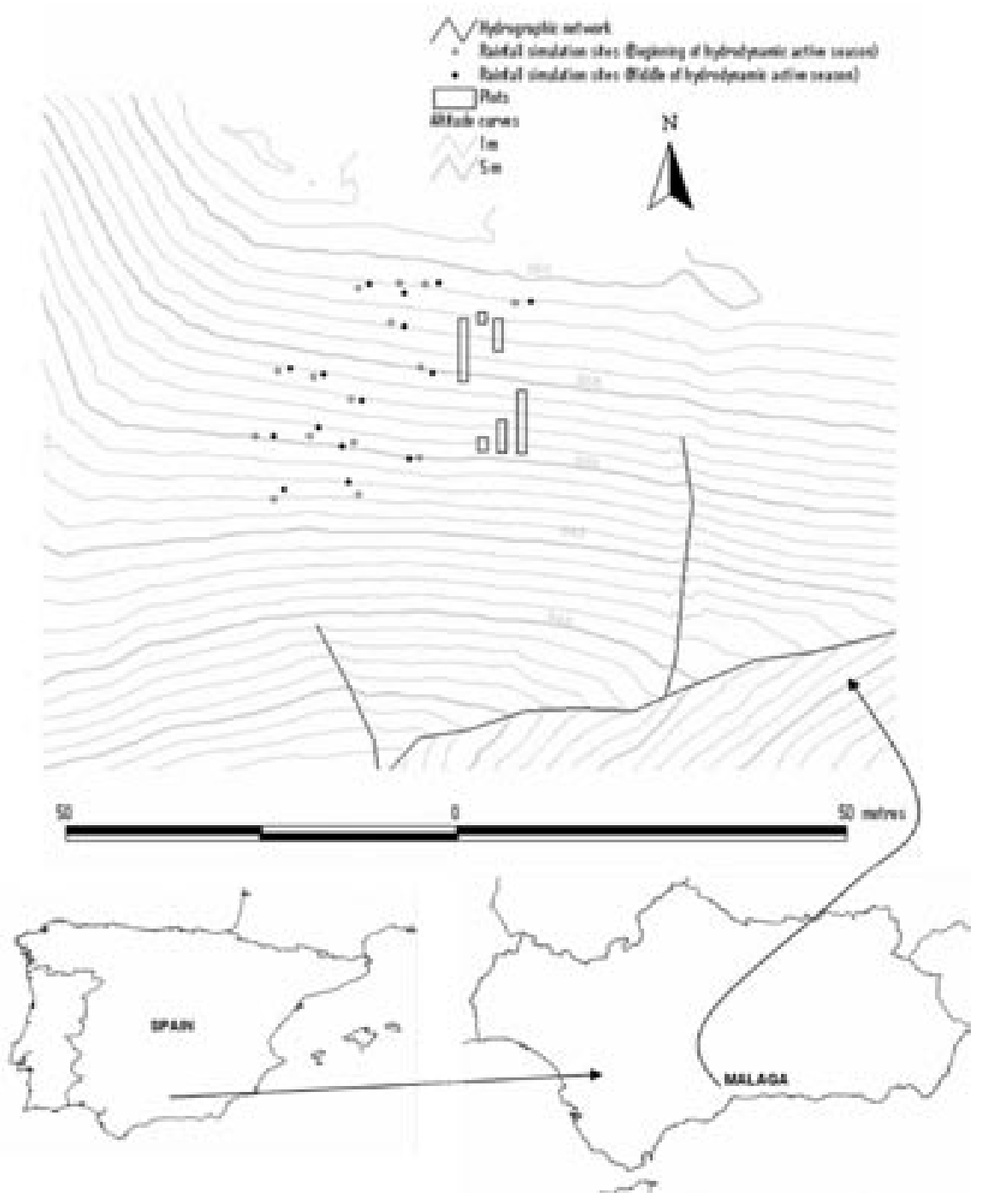




\section{2. ÁREA DE ESTUDIO}

La ladera donde se ha llevado a cabo esta investigación pertenece a los relieves que forman parte de los Montes de Málaga (Cordillera Bética, Sur de España), localizándose concretamente en la cabecera de la cuenca del arroyo Ancón, tributario del río Guadalhorce. El paisaje está dominado por la presencia de relieves de sustrato geológico metamórfico de elevadas pendientes. Estos relieves están muy afectados por la acción incisiva de una densa red drenaje la cual tiene que salvar un elevado desnivel altitudinal (generalmente $>700 \mathrm{~m}$ ) en apenas $10 \mathrm{~km}$. De este modo, la erosión hídrica es el agente geomorfológico predominante. La cubierta natural de los Montes de Málaga es muy pobre a causa de una actividad agrícola secular (olivo, almendro, vid) la cual ha sido progresivamente abandonada de modo que hoy en día coexisten campos cultivados con otros ya abandonados y recolonizados por vegetación natural, en diferentes grados según las condiciones climáticas y el grado de degradación del suelo. La consecuencia es la presencia de unas condiciones potenciales elevadas de erosión y degradación que pueden dirigir el sistema hacia la desertificación.

La ladera de la investigación es representativa del paisaje en la que se inserta y se eleva desde 535 a $563 \mathrm{~m}$ sobre el nivel del mar, su pendiente media es de $26.0 \%$ aunque oscila desde apenas un $5.0 \%$ en la cima a más de un $35.0 \%$ en la zona baja, y su exposición es igual a $175^{\circ}$. La morfología es muy similar a la del resto que caracterizan a los Montes de Málaga: el sustrato metamórfico (filitas), la elevada pendiente hacia el fondo del valle y una cima más llana definen un perfil convexo que incita a la generación de escorrentía superficial.

El régimen climático es típicamente mediterráneo con una marcada irregularidad en las precipitaciones y unas temperaturas suaves. La estación meteorológica más próxima se encuentra en la localidad de Almogía $(4 \mathrm{~km})$, a una altitud de $365 \mathrm{~m}$ s.n.m., donde la precipitación media anual es $511.1 \mathrm{~mm}$ para 48 años de registros. Como la correlación entre altitud y precipitación es muy buena en los Montes de Málaga $\left(r=0.85, \mathrm{R}^{2}=73.5 \%\right.$, Justicia-Segovia 1988), la precipitación media en la ladera sería aproximadamente igual a $590.0 \mathrm{~mm}$ y por tanto se trataría de un área en transición entre el régimen semiárido y el sub-húmedo.

La ladera puede ser un claro ejemplo de la evolución del ecosistema mediterráneo de las áreas cultivadas durante los últimos 50 años, que posteriormente fueron abandonadas y colonizadas por vegetación mediterránea de matorral. La vegetación del área corresponde a un alcornocal (Quercus suber) acompañado por un sotobosque de especies arbustivas mediterráneas en la asociación denominada Smilaci mauritanicae-Querceto rotundifoliae quer- 
cetoso suberis (Nieto-Caldera, Pérez-Latorre y Cabezudo 1990). No obstante, la vegetación de la ladera está compuesta sólo por especies de matorral mediterráneo (Cistus monpeliensis, Genita umbellata, Cistus albidus, Helichrysum retusum, Retama spaherocarpa, Ulex parviflorus). En el entorno de esta zona de vegetación natural, el uso del territorio predominante es el agrícola con cultivos de olivos y almendros. Actualmente, el pastoreo caprino suele afectar a la ladera analizada de forma esporádica.

\section{METODOLOGÍA}

La metodología se ha dividido en dos fases. La primera ha tratado de analizar el sistema eco-geomorfológico y sus elementos constituyentes en la ladera y, la segunda, medir la respuesta hidrológica y erosiva de esos elementos o micro-ambientes en dos momentos del año: a principios de otoño o comienzo de la estación activa hidrodinámica y a mediados de esta.

El análisis del sistema eco-geomorfológico se ha hecho según la metodología utilizada por Calvo et al. (2003b) adaptada a las condiciones de la ladera de la investigación. Primero, señalizamos una parcela con la misma longitud, desde la línea divisoria de aguas al talweg y con una anchura igual a $5 \mathrm{~m}$. Fotografiamos esta parcela abierta, y posteriormente fue foto-interpretada. Así, obtuvimos información sobre qué elementos o micro-ambientes eco-geomorfológicos eran más abundantes y transcendentes en la respuesta hidrológica y erosiva de la ladera. A priori esta metodología distingue tres ambientes principales: suelo desnudo, vegetación y afloramientos rocosos. Como mostramos en la tabla 1, éstos diferentes ambientes se dividen en distintas modalidades según varios criterios. Los suelos desnudos se dividen según la presencia de hojarasca o de la presencia de vegetación herbácea anual y de fragmentos rocosos en superficie. Los grupos de vegetación se distinguen según: tipo de la vegetación, densidad del follaje, cubierta, temporalidad y estado fisiológico.

La información sobre los diversos micro-ambientes que compusieron el sistema eco-geomorfológico de la ladera fue completada mediante el análisis de diferentes propiedades físicas del suelo. Tomamos muestras de suelo y las propiedades analizadas fueron las siguientes: contenido de la grava (tamizada a 2 milímetros), contenido de arcilla (metodología de la sedimentación), porosidad (picnometro), contenido de materia orgánica (contenido del carbono medido en espectrofotómetro) y estabilidad de agregados (metodología del wet sieving). 


\section{TABLA 1}

\section{AMBIENTES Y MICRO-AMBIENTES DEFINIDOS POR LA METO- DOLOGÍA DE ANÁLISIS DEL SISTEMA ECO-GEOMORFOLÓGI- CO PROPUESTA POR CALVO ET AL. (2003B).}

\begin{tabular}{|l|l|}
\hline GRUPO & SUBGRUPO \\
\hline Suelo & suelo desnudo, $<25 \%$ pedregosidad, con vegetación anual \\
\hline & suelo desnudo, $<25 \%$ pedregosidad, sin costras \\
\hline & suelo desnudo, $<25 \%$ pedregosidad, con rocas embebidas, sin costras \\
\hline & suelo desnudo, $<25 \%$ pedregosidad, con costras \\
\hline & suelo desnudo, $<25 \%$ pedregosidad, con rocas embebidas y costras \\
\hline & suelo desnudo, 25-70\% pedregosidad, con vegetación anual \\
\hline & suelo desnudo, 25-70\% pedregosidad, sin costras \\
\hline & suelo desnudo, 25-70\% pedregosidad, con costras \\
\hline & suelo desnudo, 25-70\% pedregosidad, con rocas embebidas, sin costras \\
\hline & suelo desnudo, 25-70\% pedregosidad, con rocas embebidas y costras \\
\hline & suelo desnudo, $>$ del 70\% pedregosidad \\
\hline & suelo desnudo, $>$ del 70\% pedregosidad, con rocas embebidas \\
\hline Afloramientos rocosos \\
\hline Vegetación & vegetación arbórea \\
\hline & vegetación de matorral de alta densidad \\
\hline & vegetación de matorral de baja densidad \\
\hline & vegetación herbácea anual \\
\hline & vegetación seca, conectadas con el suelo \\
\hline
\end{tabular}

Finalmente, el comportamiento hidrológico ha sido analizado mediante dos tipos de aproximaciones. En primer lugar, llevamos a cabo simulaciones de lluvia en los micro-ambientes representativos del sistema eco-geomorfológico al principio de la estación hidrodinámica activa (Sept/Oct 2004) y a mediados de esta (marzo de 2005). Para la estimación de la escorrentía hemos usado el simulador de precipitación diseñado por Calvo et al. (1988). Durante la simulación de la precipitación, esta mantuvo valores de intensidad similares a las intensidades del área del estudio que pueden ser medidas: 36.0 a 40.0 mm h-1 durante períodos de 15 minutos (Martínez-Murillo 2006). La metodología usada para realizar las simulaciones ha sido la siguiente: i) colocación de las micro-parcelas según los micro-ambientes analizados del sistema ecogeomorfológico, tomando fotografías de ellas y midiendo algunas características (pendiente, cubierta vegetal y pedregosidad superficial), ii) inserción de las sondas de TDR-Tektronik en el suelo para la medición del contenido de humedad, a 5, 10 y $15 \mathrm{~cm}$ de profundidad, y iii) los experimentos tenían 
una duración de 40 minutos distribuidos de la manera siguiente: 20 minutos

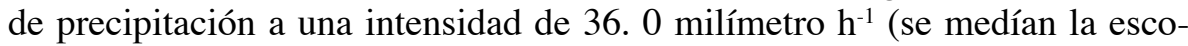
rrentía, los sedimentos y la humedad del suelo), 5 minutos sin precipitación (solamente la humedad del suelo era medida), 10 minutos de precipitación a la misma intensidad (escorrentía, sedimentos y humedad del suelo) y de nuevo, 5 minutos sin precipitación (solamente humedad del suelo). A partir del inicio de la escorrentía, esta fue medida cada minuto, los sedimentos cada cuatro o cinco minutos y la humedad del suelo cada dos minutos. Más adelante en laboratorio las muestras de sedimentos fueron secadas a $105^{\circ} \mathrm{C}$ en una estufa para relacionarlo con el volumen de escorrentía y así calcular la concentración de sedimentos.

Al mismo tiempo, y en segundo lugar, a partir del 1 de Septiembre 2004 hasta el 30 Abril de 2005, el comportamiento hidrológico y erosivo del sistema eco-geomorfológico fue analizado también mediante el seguimiento de la evolución de la escorrentía y el transporte de sedimentos en parcelas cerradas. Estas parcelas tienen distintas longitudes: dos parcelas de $1.5 \mathrm{~m}$ de longitud, dos parcelas de $4 \mathrm{~m}$ longitud, y dos parcelas de 8 metros de longitud y todas de $1 \mathrm{~m}$ de anchura. Las parcelas eran representativas del sistema eco-geomorfológico de la ladera.

\section{RESULTADOS}

\subsection{Micro-ambientes del sistema eco-geomorfológico de la ladera}

Siguiendo la metodología aplicada por Calvo et al. (2003) hemos definido tres ambientes en la ladera: matorral de alta densidad (HDS), matorral de baja densidad (LDS) y suelo con vegetación herbácea (SHV).

La unidad de HDS está definida por especies vegetales de alta densidad de hojas y cubierta vegetal: Quercus coccifera, Genista umbellata, Chamaerops humilis y Helichrysum stoechas, éste último dependiendo de la estación del año. El suelo tiene un espesor superior a 25 centímetros. El horizonte A tiene un alto contenido en materia orgánica $(>4.5 \%)$ y el B -cámbico- en gravas y estructura sub-angular. La textura del suelo es generalmente franco o francoarcillosa debido a un mayor contenido de limos. Estas condiciones del suelo implican a priori una importante capacidad de retención hidrica. La superficie del suelo está ocupada por un alto porcentaje de fragmentos de rocas $(25$ a $70 \%$ en $1 \mathrm{~m}^{2}$ ) acompañados por abundantes restos vegetales.

La unidad SHV corresponde a las áreas de ladera de suelo cubierto por vegetación herbácea anual -terófitas-, y zonas de fragmentos rocosos $(>70 \%$ en $1 \mathrm{~m}^{2}$ ) no embebidos. La vegetación herbácea es la consecuencia del ciclo 
pluviométrico anual. Sin embargo, el suelo no queda totalmente descubierto en verano porque una parte de esa vegetación herbácea seca continúa sobre él. Algunos patches de esta unidad corresponden a las micro-terrazas originadas por el paso de ganado, dando lugar a zonas de terreno de menor pendiente a mitad de ladera $(<10 \%)$. Generalmente, el suelo corresponde a un Cambisol pero donde ha estado muy afectado por la erosión hídrica, su espesor y horizontes definen un Regosol. El suelo tiene un contenido bajo de materia orgánica $(<2.0 \%)$, la textura es franco-arcillo-arenosa en los primeros centímetros pero el contenido de la arcilla es muy similar a las otras unidades por debajo de los 5 centímetros. La estructura esta menos desarrollada y la estabilidad de los agregados no alcanza en húmedo el $60 \%$. Un mayor contenido en arenas junto a la disposición de la pedregosidad en superficie origina unas condiciones más favorables para la infiltración del agua en el suelo. Sin embargo, esta puede verse reducida en profundidad, de $5 \mathrm{a} 10 \mathrm{~cm}$, debido al incremento del contenido de la arcilla del suelo de esta unidad.

La unidad LDS incluye las especies vegetales con una densidad de hojas menor como Cistus monpeliensis, Cistus albidus, Lavandula stoechas, Retama sphaerocarpa y Phlomis purpurea aunque algunos de estos pueden cambiar su densidad durante la estación de lluvias como Cistus monpeliensis. Las características del suelo son intermedias entre las otras unidades. La materia orgánica es muy similar a la unidad HDS ( 3 a 5\%) y solamente la cubierta de fragmentos rocosos y la textura es igual que en el suelo de la unidad de SHV: $>70 \%$ y franco-arcillo-arenoso en superficie y franco-arcilloso en profundidad.

En general, el patrón de vegetación de la ladera permite observar estas tres unidades a lo largo de toda su longitud. No obstante, podemos definir ciertas tendencias: las unidades de matorral HDS y LDS se localizan preferentemente en el tercio superior e inferior de la ladera (hacia la cima y el talweg), mientras que la unidad de suelo SHV lo hace preferentemente en la zona media de la parcela.

En cualquier caso, estaríamos ante una ladera heterogénea en relación a los diferentes elementos que componen las relaciones suelo-agua-planta, lo que podría ser causa y efecto de su complejo comportamiento hidrológico.

\subsection{Variaciones de la escorrentía y la emisión de sedimentos}

Para determinar el comportamiento hidrológico de los microambientes llevamos a cabo simulaciones de precipitación atendiendo al procedimiento ya descrito. 


\subsubsection{Inicio de la estación hidrodinámica activa}

La estación hidrodinámica activa comienza con el inicio de las precipitaciones tras el período estival que caracteriza al clima mediterráneo. La vegetación se encuentra en un momento de estrés hídrico y como consecuencia ha disminuido su actividad fisiológica durante el verano. El contenido en agua del suelo es generalmente inferior al $5 \%$ en las tres unidades definidas. La permanencia de estas condiciones a lo largo del verano supone una reducción de la cubierta vegetal pues esta no ha tenido garantizada la disponibilidad de agua: si consideramos conjuntamente la cubierta ofrecida por el matorral y la cubierta herbácea, esta se redujo al $46 \%$ a lo largo de la estación seca, partiendo de una cubierta máxima del 75 u 80\% al final de la estación hidrodinámica activa. El suelo mostraba un aspecto compactado a causa del contenido tan bajo en agua pues la ausencia de esta supone una reducción de su volumen (Kutilek y Nielsen 1994).

Las simulaciones de lluvia realizadas al principio de la estación hidrodinámica activa demuestran lo que podría suceder con las primeras precipitaciones del otoño: las tres unidades pueden emitir escorrentía (figura 2). Sin embargo, hay diferencias entre ellas porque la escorrentía no se genera de la misma forma. La unidad HDS ha emitido escorrentía en un experimento, LDS en dos experimentos y SHV en tres experimentos como mostramos. La evolución de la humedad del suelo la mostramos en la figura 3.

El funcionamiento hidrológico de la unidad HDS ha sido infiltrador. No obstante, se observó generación de escorrentía en una simulación de lluvia completa y en la mitad de otra. Estos dos experimentos se caracterizaron por un menor volumen de escorrentía en la primera parte y mayor en la segunda debido a la reducción de la capacidad de infiltración del suelo. También ha sido característica la reducción de la emisión de sedimentos a lo largo de todo el experimento. Así, incluso con una elevada cubierta vegetal, en la simulación HDS-3 la escorrentía comienza en apenas 5 min. y se mantiene por encima de los $10 \mathrm{~mm} \mathrm{~h}^{-1}$. Esta escorrentía se caracterizó por una concentración en sedimentos superior a $2.0 \mathrm{~g} \mathrm{~L}^{-1}$. Respecto a la evolución de la humedad del suelo, suele incrementarse trascurridos unos diez minutos desde el inicio a $5 \mathrm{~cm}$. En esta profundidad se observan dos comportamientos al final del experimento: suelos con más del $20 \%$ de humedad y suelos con menos del $20 \%$. A $10 \mathrm{~cm}$ el contenido de humedad nunca ha superado el nivel de $10 \%$.

La unidad LSD ha generado escorrentía en dos de los experimentos llevados a cabo coincidiendo con las micro-parcelas de mayor pendiente. Pero el volumen de escorrentía emitido ha sido superior que en la unidad anterior (en general $>15.0 \mathrm{~mm} \mathrm{~h}^{-1}$ ). La escorrentía se ha iniciado más tarde (LDS-3, 8 min., y LDS-5, $11 \mathrm{~min}$.) y en las micro-parcelas de menor contenido de humedad 
en el suelo. Los sedimentos arrastrados han sido superiores a la unidad anterior también. No obstante, se ha observado el mismo patrón temporal: menos escorrentía y más sedimentos en la primera parte, y más escorrentía y menos sedimentos en la segunda. La evolución del contenido de humedad durante la simulación es muy variable pero se observa un incremento lento y escaso en la primera parte del experimento, y algo superior en la segunda.

Finalmente, la unidad SHV ha tenido un funcionamiento hidrológico con mayor tendencia a la generación de escorrentía (4 de 5 experimentos). Sin embargo, al contrario que las otras unidades, ha comenzado a emitir escorrentía algo más tarde (7 a 12 minutos) y en general con un volumen menor. Pero hay que apuntar que en momentos puntuales esta emisión de flujo superficial ha sido más agresiva (SHV-5). También las concentraciones de sedimentos han sido inferiores a pesar de la falta de cubierta de matorral, oscilando de 1 a $2 \mathrm{~g}$ $\mathrm{L}^{-1}$. La única micro-parcela que ha quedado sin emitir escorrentía, se situaba sobre una micro-terraza de pastoreo (terreno de menor pendiente). La humedad del suelo ha aumentado más en la primera parte ante un suelo más seco y se mantiene estable en la segunda pero siempre aumentando. 


\section{FIGURA 2}

EMISIÓN DE ESCORRENTÍA Y DE SEDIMENTOS DURANTE LAS SIMULACIONES DE PRECIPITACIÓN AL PRINCIPIO DE LA ESTACIÓN HIDRODINÁMICA ACTIVA.
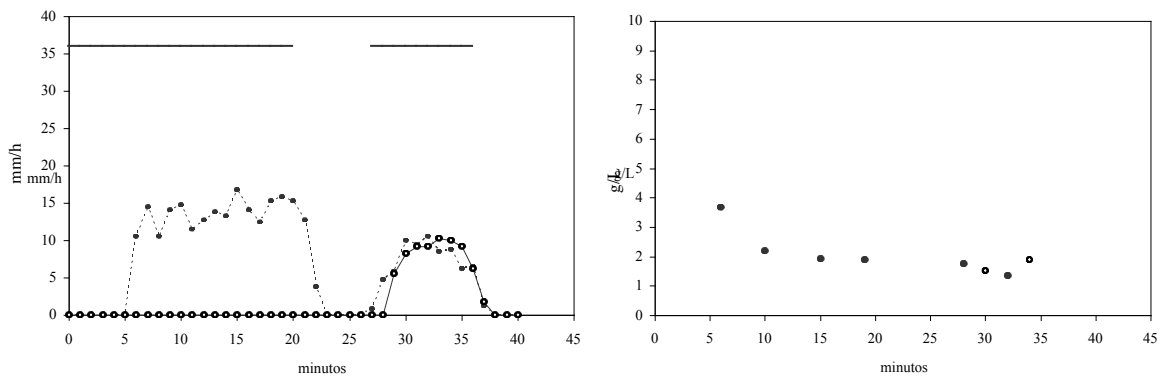

— Intensidad ........ HDS-3 - — HDS-4
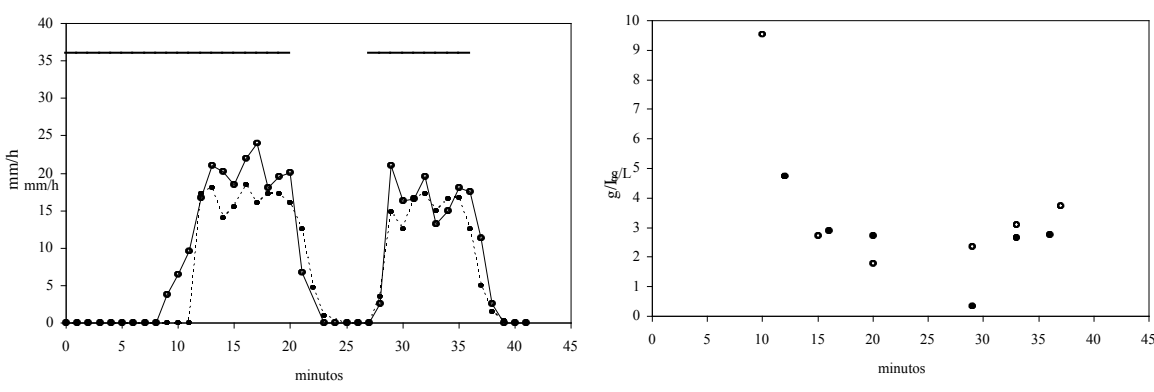

- Intensidad $\cdots$..... LDS-3 $\longrightarrow$ LDS-5

- LDS-3 O LDS-5
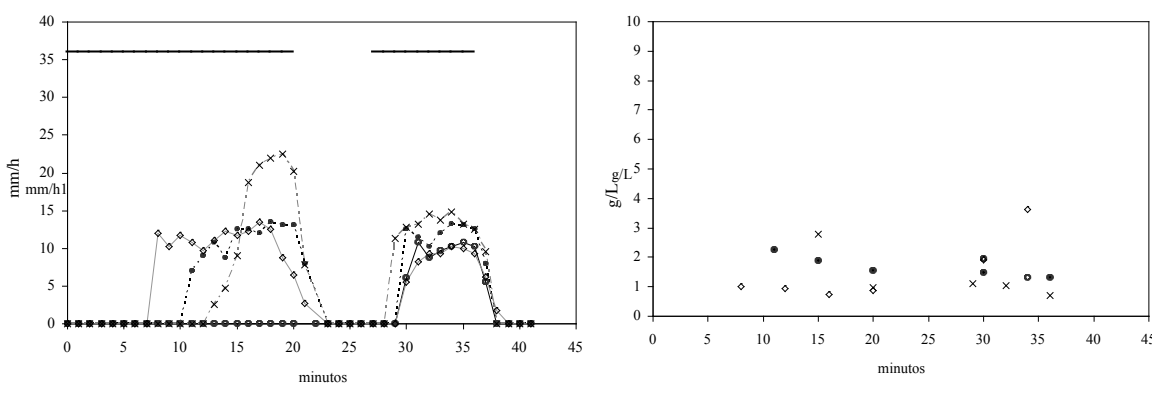

—Intensidad $\multimap$ SHV-1 $\ldots \ldots$ SHV-2 0 SHV-4 $\ldots$ - * - SHV-5 
FIGURA 3

EVOLUCIÓN DEL CONTENIDO EN AGUA DEL SUELO DURANTE LOS EXPERIMENTOS DE LA SIMULACIÓN DE LA PRECIPITACIÓN EN PRINCIPIO DE LA ESTACIÓN HIDRODINÁMICA ACTIVA.

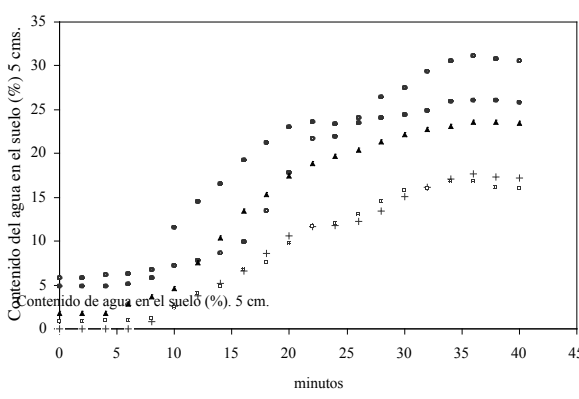

- HDS- 1 a HDS- 2 . HDS-3 + HDS- 4 a HDS-5

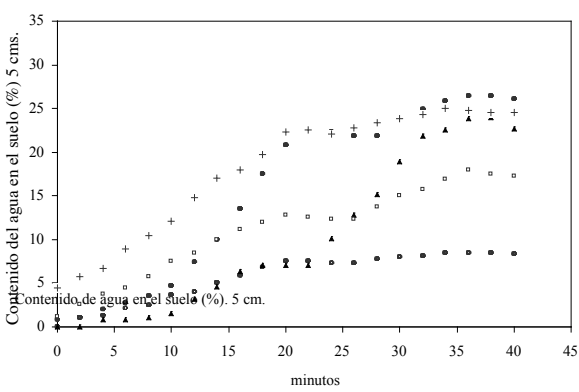

. LDS-1 o LDS-2 „ LDS-3 + LDS-4 a LDS-5

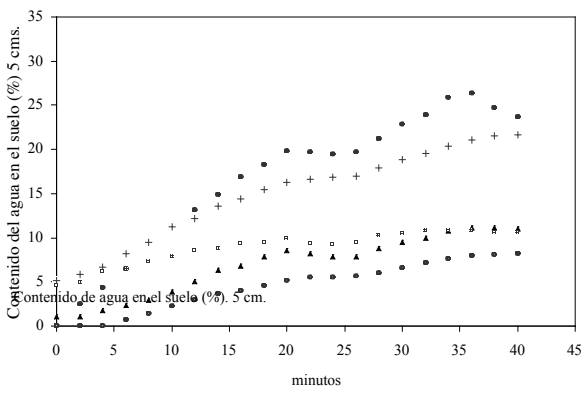

- SHV-1 口 SHV-2, SHV-3 + SHV-4, SHV-5

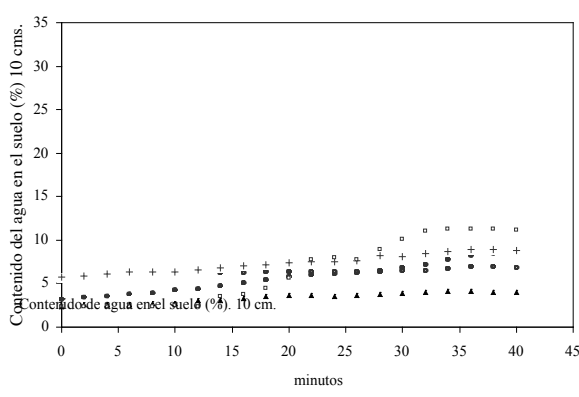

- HDS- 1 .HDS- 2 А HDS-3 + HDS- 4 a HDS- 5

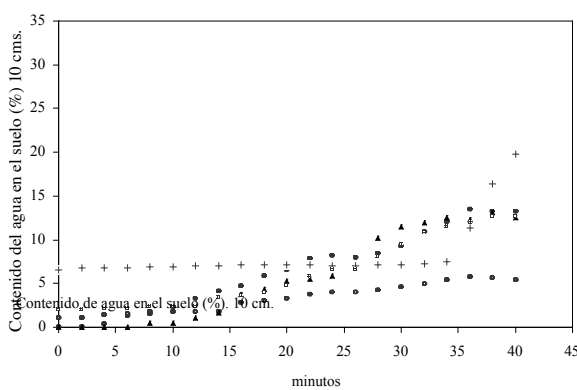

. LDS-1 a LDS-2 ^ LDS-3 + LDS-4 . LDS-5

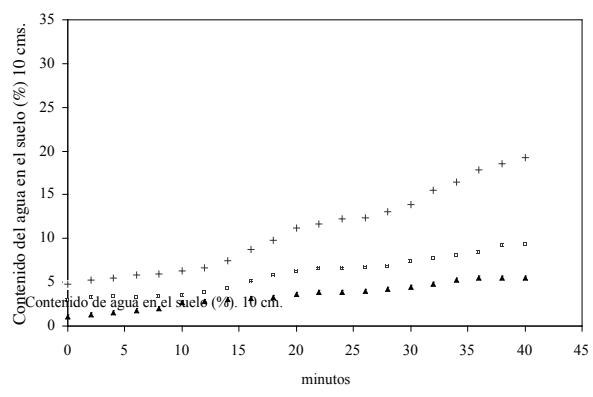

. SHV-3 + SHV-4 . SHV-5

En definitiva, al inicio de la estación hidrodinámica activa, la ladera puede llegar a generar escorrentía en gran parte de su extensión, adquiriendo un comportamiento hidrológico acorde con el modelo hidrológico de Horton (1933). 


\subsubsection{Durante la estación hidrodinámica activa}

Como consecuencia del paso de varios eventos lluviosos desde el comienzo de esta estación en el otoño, la vegetación recupera un estado óptimo a causa del aumento de la disponibilidad de agua en suelo. Esto produce un aumento de la densidad foliar en el matorral y el crecimiento de nueva vegetación herbácea anual y por tanto se incrementa la cubierta del suelo. El suelo ha perdido el aspecto compacto de la estación seca y a priori parece más susceptible a la infiltración del agua. En el momento de las ejecuciones de los experimentos, el contenido de agua del suelo estaba siempre alrededor del $8.0 \%$, excepto en dos micro-parcelas con 6.1 y $7.6 \%$. La principal diferencia entre el comportamiento hidrológico al principio y a mediados de la estación hidrodinámica es que sólo se genera escorrentía en la unidad SHV. Pero incluso en esta, se registra cuando la pendiente excedió del 20\%. Las unidades HDS y LDS infiltraron siempre a lo largo de todos los experimentos llevados a cabo con la misma intensidad.

En las figuras 4 y 5 mostramos la evolución de la concentración de la escorrentía, del sedimento y del contenido en agua del suelo. Respecto a la inicio de la estación hidrodinámica, el inicio de la escorrentía sucede antes en la primera parte del experimento debido a un contenido de agua en el suelo más alto. Las micro-parcelas comienzan a emitir escorrentía cuando la humedad del suelo alcanza un contenido cercano al 15\%. El inicio del flujo superficial tiene lugar más tarde en la segunda parte del experimento respecto a las simulaciones anteriores de precipitación en la unidad de SHV. Los volúmenes de escorrentía de SHV tienden a ser más bajos en estos momentos no excediendo demasiado los $10 \mathrm{~mm} \mathrm{~h}^{-1}$ en la primera y segunda fase. Ambas fases presentan una evolución muy similar en la escorrentía, incluso estando el suelo más húmedo en la segunda que en la primera. La concentración de sedimentos es siempre menor de $2.5 \mathrm{~g} \mathrm{~L}^{-1}$ y disminuye desde el comienzo de la emisión de escorrentía hasta el final cuando se registra el transporte más bajo de partículas finas. 
FIGURA 4

ESCORRENTÍA Y EMISIÓN DE SEDIMENTOS DURANTE LAS

SIMULACIONES DE LA PRECIPITACIÓN A MEDIADOS DE LA

ESTACIÓN HIDRODINÁMICA ACTIVA
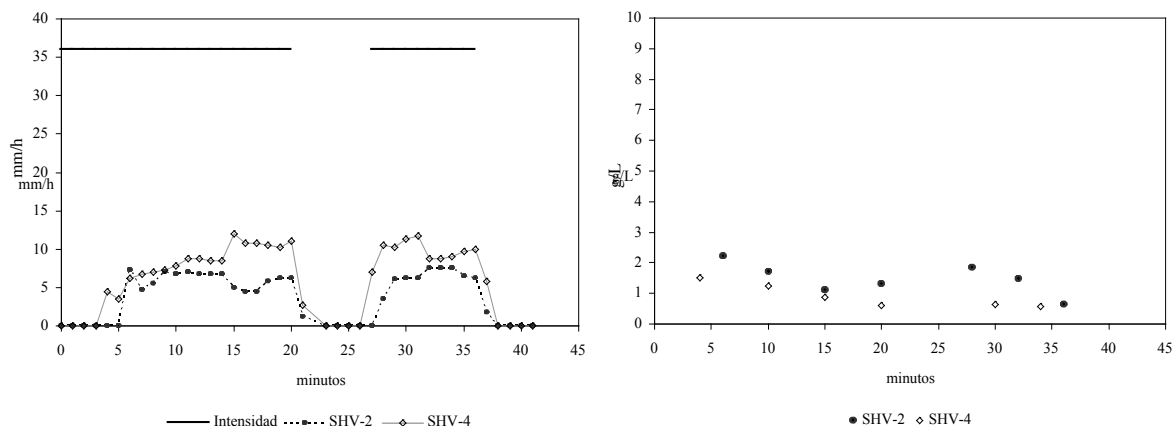

Casi todas las unidades han podido superar el $20 \%$ de contenido de agua. Sin embargo, ha habido alguna micro-parcela que siempre ha permanecido por debajo del $15 \%$ en la profundidad de 5 centímetros debido a la existencia de factores que reducían la capacidad de infiltración del agua: una densidad más elevada de densidad foliar en HDS la cual retrasó la llegada del agua a la superficie del suelo, y en las unidades LDS y SHV una pendiente más alta que limitó la capacidad de la infiltración del suelo. La unidad SHV registró los contenidos de agua en el suelo mayores a 5 centímetros, seguidas por las unidades LDS y HDS en este orden, incluso siendo la única unidad que emitió escorrentía. El comportamiento del contenido de agua del suelo es muy similar a $10 \mathrm{~cm}$, siendo alcanzado el contenido más alto en las unidades LDS y SHV. La unidad HDS muestra dos comportamientos: uno, que consigue alcanzar claramente un contenido del $20 \% \mathrm{y}$, otro, que permanece alrededor del $10 \%$, muy estable coincidiendo en la micro-parcela con la presencia de Genista umbellata, especie vegetal de gran densidad. 


\section{FIGURA 5}

EVOLUCIÓN DEL CONTENIDO EN AGUA DEL SUELO DURANTE LOS EXPERIMENTOS DE SIMULACIÓN DE PRECIPITACIÓN A MEDIADOS DE LA ESTACIÓN HIDRODINÁMICA ACTIVA

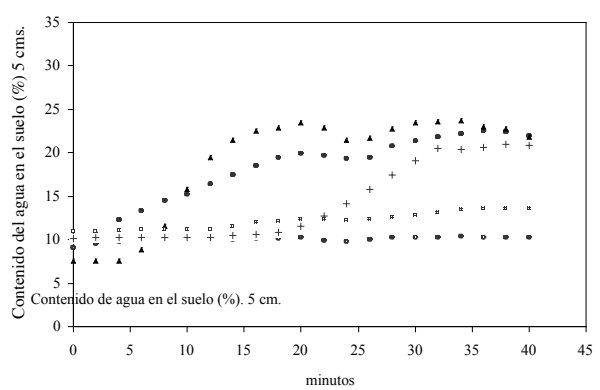

. HDS-1 a HDS-2 ^HDS-3 + HDS-4 •HDS- 5

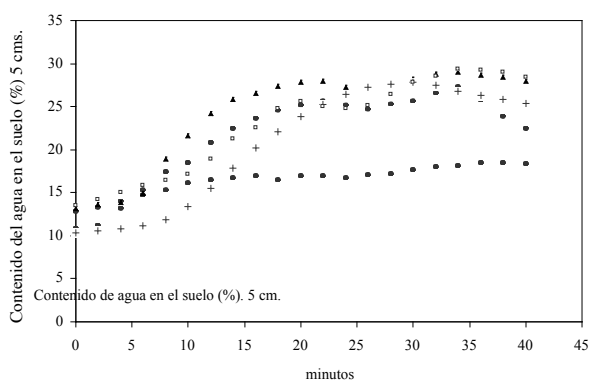

- LDS-1 LDS-2 . LDS-3 + LDS-4 LDS-5

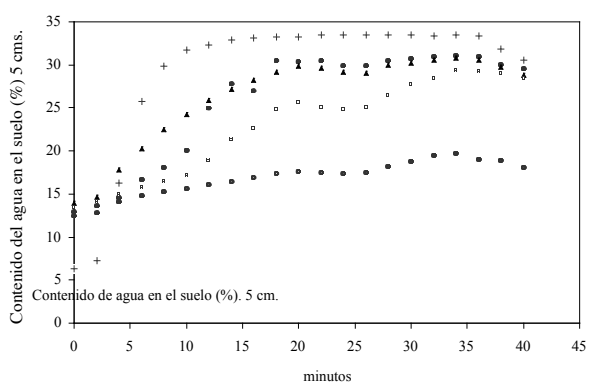

- SHV-1 a SHV-2 . SHV-3 + SHV-4 。 SHV-5

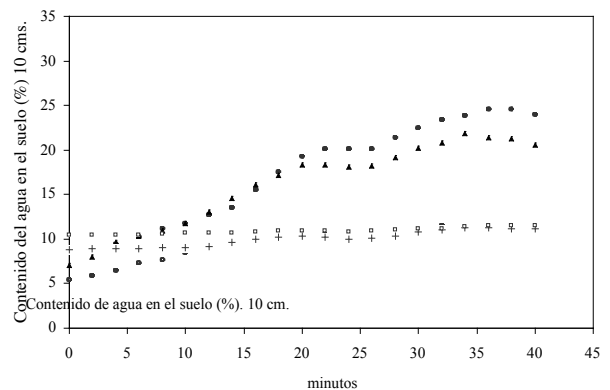

- HDS-1 。 HDS-2 . HDS-3 + HDS-4 a HDS-5

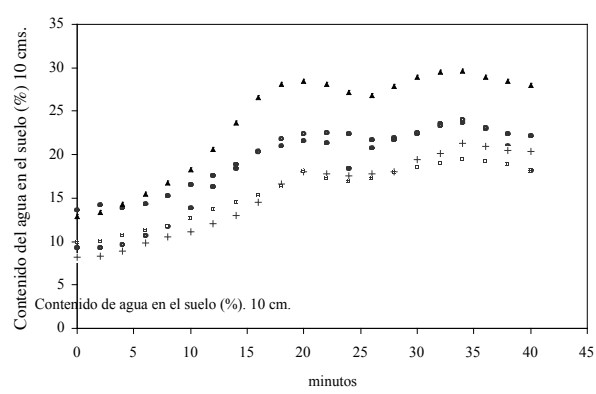

- LDS-1 a LDS-2 ^ LDS-3 + LDS-4 。 LDS-5

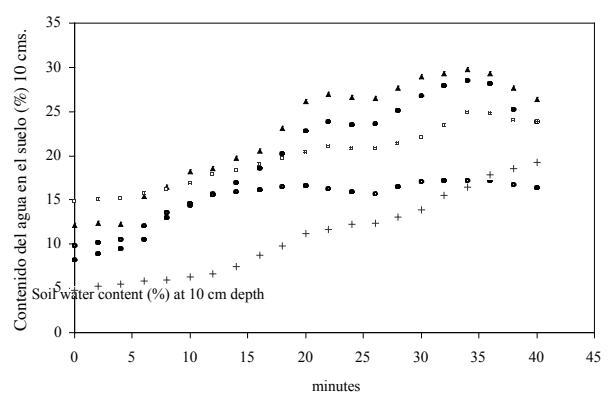

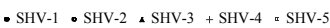


La ladera se comporta desde el punto de vista hidrológico como un área en la que predominan los procesos de infiltración. Las zonas de matorral actúan como sumideros de precipitación así como las zonas de suelo cubierto por vegetación herbácea anual, salvo aquellas en las que la elevada pendiente lo impide. Entonces estamos ante unos mecanismos de generación de escorrentía basados en la saturación del perfil edáfico.

\subsection{Determinación de la conectividad hidrológica: las parcelas cerradas}

La tabla 2 muestra las características físicas de las parcelas. A pesar de que cada una tiene una longitud diferente, en términos relativos están compuestas por características similares, principalmente en lo que a las unidades de suelo se refiere. Como se puede observar, la unidad SHV se ha dividido en tres niveles porque observamos que había leves diferencias que podrían tener consecuencias hidrológicas y erosivas.

TABLA 2

PRINCIPALES CARACTERÍSTICAS FÍSICAS DE LAS PARCELAS

\begin{tabular}{|l|c|c|c|c|c|c|}
\hline Parcelas & $1.5 \mathrm{a}$ & $1.5 \mathrm{~b}$ & $4 \mathrm{a}$ & $4 \mathrm{~b}$ & $8 \mathrm{a}$ & $8 \mathrm{~b}$ \\
\hline Longitud (m) & 1.5 & 1.5 & 4.0 & 4.0 & 8.0 & 8.0 \\
\hline Desnivel (m) & 0.30 & 0.40 & 1.00 & 1.20 & 1.85 & 2.30 \\
\hline Pendiente (\%) & 20.0 & 26.7 & 25.0 & 30.0 & 23.1 & 28.8 \\
\hline Orientación ( $)$ & 185.0 & 185.0 & 185.0 & 185.0 & 185.0 & 185.0 \\
\hline Cubierta vegetal (\%) & 50.0 & 40.0 & 20.0 & 40.0 & 50.0 & 40.0 \\
\hline VAD (\%) & 20.0 & 85.0 & 70.0 & 80.0 & 30.0 & 75.0 \\
\hline VBD (\%) & 80.0 & 15.0 & 30.0 & 20.0 & 70.0 & 25.0 \\
\hline Suelo (\%) & 50.0 & 60.0 & 80.0 & 60.0 & 50.0 & 60.0 \\
\hline Suelo 1 (\%) $(*)$ & 15.0 & 10.0 & 5.0 & 15.0 & 20.0 & 25.0 \\
\hline Suelo 2 (\%) $(*)$ & 55.0 & 55.0 & 65.0 & 60.0 & 55.0 & 55.0 \\
\hline Suelo (3) $(*)$ & 30.0 & 35.0 & 20.0 & 25.0 & 25.0 & 20.0 \\
\hline
\end{tabular}

(*): Suelo 1, Suelo $>70 \%$ pedregosidad; Suelo 2, Suelo $>70 \%$ pedregosidad, hojarasca y vegetación herbácea; Suelo 3, idem anteriores pero de menor pendiente (acumulación). 
FIGURA 6

VOLUMEN DE PRECIPITACIÓN Y EVOLUCIÓN DE LA PRECIPITACIÓN ACUMULADA EN LA LADERA DURANTE EL PERIODO DE TIEMPO DE SEGUIMIENTO DE LAS PARCELAS CERRADAS

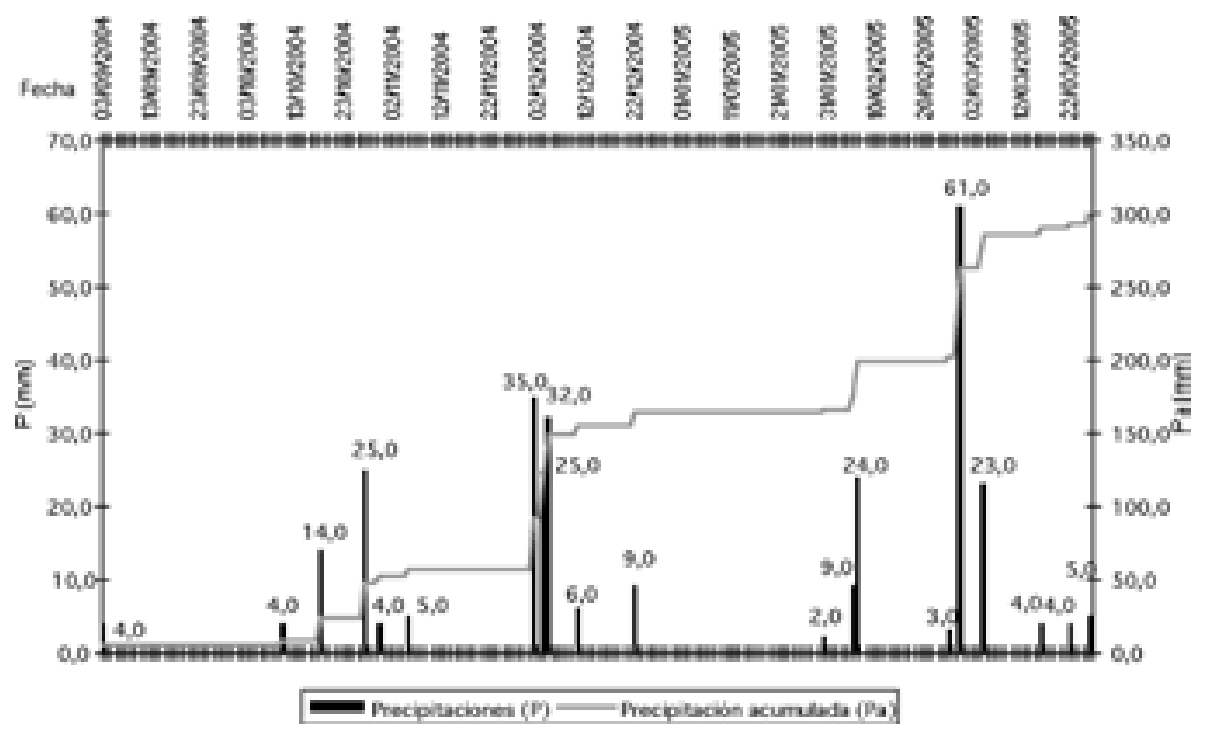

\subsubsection{Eventos pluviométricos}

Desde 09/01/2004 a 04/30/05 recogimos en el pluviómetro colocado en la ladera un volumen total igual a $298.0 \mathrm{~mm}$ en 30 días de precipitaciones, muy escasa precipitación para la estación del año a la que se refiere. Dicha cantidad tan solo supone un total de precipitación casi un 36\% menos que el valor medio para el mismo período de tiempo (444 mm en la estación de Almogía). Los eventos de precipitación han oscilado desde 2.0 a $61.0 \mathrm{~mm}$. (figura 6). Aquellos cuya precipitación ha sido inferior a $10.0 \mathrm{~mm}$ han sido muy numerosos y aún más los inferiores de $5.0 \mathrm{~mm}$. como consecuencia del tiempo predominante de vientos de dirección E y SE, como se observaron en los boletines oficiales del Instituto Meteorológico Nacional y que no proporcionan generalmente grandes volúmenes de las precipitaciones (Senciales-González 1996). Las precipitaciones más cuantiosas se han concentrado en cuatro eventos: 25.0 de octubre, $35.0,32.0$ y 25.0 de diciembre, 24.0 de febrero, y 61.0 de marzo.

Siendo estrictos con la definición de la estación activa hidrodinámica, el sistema presentó una actividad hidrológica muy pobre durante el período 
mencionado a causa de la estación húmeda inusual. Sin embargo, algunos eventos de precipitación fueron suficientes para producir escorrentía y transporte de sedimentos.

TABLA 3

RESUMEN DE LAS CARACTERÍSTICAS PRINCIPALES DE LA ESCORRENTÍA Y EL TRANSPORTE DE SEDIMENTOS DE LAS PARCELAS CERRADAS

\begin{tabular}{|l|c|c|c|c|c|c|}
\hline & \multicolumn{6}{|c|}{ Parcela } \\
\hline q (mm m-2) & $1,5 \mathrm{a}$ & $1,5 \mathrm{~b}$ & $4 \mathrm{a}$ & $4 \mathrm{~b}$ & $8 \mathrm{a}$ & $8 \mathrm{~b}$ \\
\hline Media & 0,22 & 0,04 & 0,34 & 0,37 & 0,19 & 0,15 \\
\hline Ds & 0,67 & 0,11 & 0,95 & 1,16 & 0,44 & 0,50 \\
\hline máx & 3,00 & 0,47 & 4,19 & 5,14 & 1,81 & 2,25 \\
\hline mín & 0,00 & 0,00 & 0,00 & 0,00 & 0,00 & 0,00 \\
\hline CV & 312,06 & 263,47 & 280,81 & 313,45 & 231,23 & 326,41 \\
\hline qa & 4,31 & 0,82 & 6,74 & 7,42 & 3,78 & 3,08 \\
\hline Cs (g 1-1) & $1,5 \mathrm{a}$ & $1,5 \mathrm{~b}$ & $4 \mathrm{a}$ & $4 \mathrm{~b}$ & $8 \mathrm{a}$ & $8 \mathrm{~b}$ \\
\hline Media & 0,35 & 0,24 & 0,08 & 0,09 & 0,15 & 0,12 \\
\hline Ds & 0,33 & 0,47 & 0,11 & 0,16 & 0,25 & 0,22 \\
\hline máx & 1,21 & 1,63 & 0,34 & 0,61 & 0,99 & 0,86 \\
\hline mín & 0,00 & 0,00 & 0,00 & 0,00 & 0,00 & 0,00 \\
\hline CV & 93,97 & 197,18 & 142,56 & 177,20 & 167,09 & 189,94 \\
\hline Sa & 1,53 & 1,20 & 3,17 & 4,34 & 7,14 & 4,80 \\
\hline
\end{tabular}

Abreviaturas: q, escorrentía; Cs, concentración de sedimentos; Ds, desviación estándar; máx, valores máximos; mín, valores mínimos, CV, coeficiente de variación; qa, escorrentía acumulada; Sa, peso de sedimentos acumulados.

\subsubsection{Escorrentía y arrastre de sedimentos}

Los valores medios no han sido muy altos (tabla 3 ) comparados con los resultados obtenidos en las micro-parcelas en las simulaciones de precipitación y mostramos la evolución de estas variables en la figura 7. 


\section{FIGURA 7}

PRECIPITACIÓN, ESCORRENTÍA Y TRANSPORTE DE SEDIMENTOS EN LAS PARCELAS CERRADAS DE DIFERENTES LONGITUDES DESDE EL 09/01/2005 AL 04/30/2005
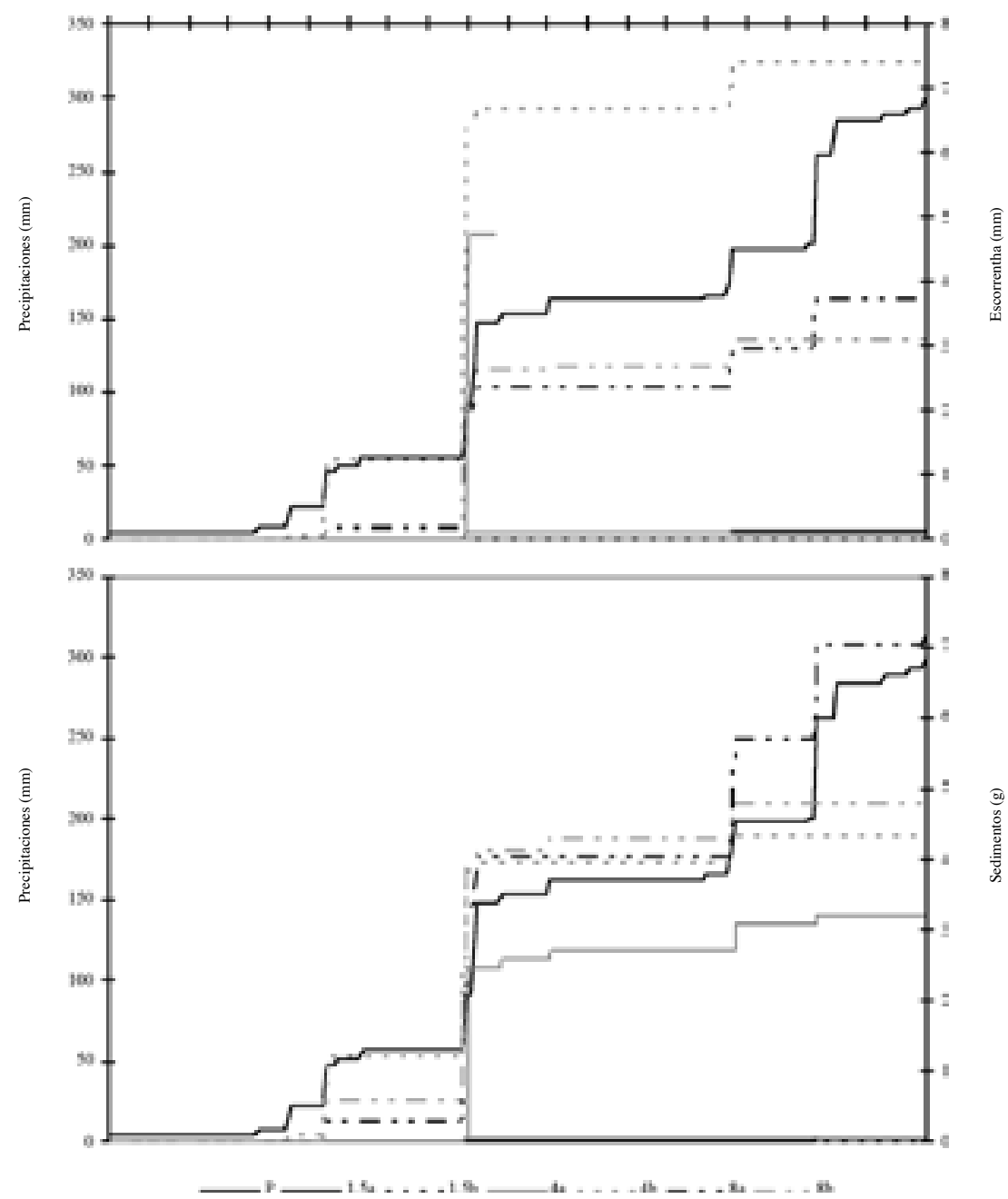
Hay diferencias entre los eventos que generaron el valor máximo de escorrentía y aquellos que produjeron los máximos de concentración de sedimentos: la escorrentía más elevada fue alcanzada el 30 de Noviembre durante una precipitación de $35.0 \mathrm{~mm}$, mientras que la concentración máxima de sedimentos tuvo lugar en varios eventos de diferentes volúmenes, aunque inferiores al anterior y que parecen haber sido más intensos al arrastrar más sedimentos, con menos volumen de escorrentía.

La escorrentía no ha seguido un patrón similar a las precipitaciones. No todos los eventos pluviométricos generaron escorrentía. Hasta diciembre podemos observar dos comportamientos: uno, corresponde a los eventos que generan escorrentía (parcela $1.5 \mathrm{~b}, 8 \mathrm{a}$ y $8 \mathrm{~b}$ ) y otro, a los que no producen flujo superficial (parcelas 1.5a, 4a y 4b).

Diciembre fue un mes con varios y abundantes eventos. Las parcelas de cuatro metros fueron las que registraban más volumen de escorrentía. Otras parcelas apenas si generaron escorrentía y por tanto no emitieron sedimentos, como ocurrió en $1.5 \mathrm{~b}, 8 \mathrm{a}$ y $8 \mathrm{~b}$. Los eventos pluviométricos de diciembre sirven para diferenciar distintos comportamientos respecto a la erosión hídrica.

En marzo, solo en la parcela 8a hay un fuerte aumento en el transporte de sedimento durante los eventos ocurridos. En estos momentos, al contrario que la escorrentía, el comportamiento de las parcelas con la misma longitud tiende a ser similar en las parcelas de cuatro y ocho metros. Los sedimentos acumulados también parecen estar relacionados con la longitud de las parcelas pues al aumentar la longitud, los sedimentos también lo hacen. Solo la parcela 8a mantiene niveles similares a las parcelas de cuatro metros. Si los sedimentos exportados aumentan con la longitud y la escorrentía no, la densidad del flujo debe variar igualmente.

Por tanto, si comparamos la evolución temporal de la escorrentía y de los sedimentos, observamos que: i) hasta los eventos de diciembre ambas variables se mantienen cercanas y la concentración de sedimentos es más alta en $1.5 \mathrm{a}, 8 \mathrm{a}$ y $8 \mathrm{~b}$, y ii) después los eventos de diciembre, el flujo superficial tiende a ser menos denso pues la curva de la escorrentía crece más que la de concentración. Esta evolución es el resultado de las consecuencias del crecimiento de la vegetación anual herbácea durante esa primera parte de la estación hidrodinámica activa puesto que actúa como protección de la superficie del suelo contra el impacto de la gota (unido a la alta cubierta de fragmentos de roca) y filtrado la escorrentía que circula ladera abajo con sedimentos en suspensión.

La figura 8 representa los valores estimados y observados de escorrentía total de las parcelas de 4 y $8 \mathrm{~m}$, calculado a partir de la premisa que las parcelas de $4 \mathrm{~m}$ deben haber emitido 2.7 veces más volumen de escorrentía que las de 1.5 y las de $8 \mathrm{~m}$, hasta 5.3 veces más. Los valores estimados tienden a ser mayores que los observados lo que manifiesta el comportamiento hi- 
drológico complejo del sistema como consecuencia de la interacción con los diversos ambientes eco-geomorfológicos incluidos en las parcelas cerradas. Las relaciones entre la longitud y la emisión de escorrentía y de sedimentos han sido calculadas mediante las correlaciones de Pearson. El mejor resultado corresponde a la relación entre longitud y transporte de sedimento $(r=0.52$. $\mathrm{p}<0.050)$. La escorrentía solo obtiene un resultado aceptable si la comparamos con el volumen de precipitación $(r=0.45$. p<0.050). También, las dos variables hidrológicas se han comparado con diferentes rasgos eco-geomorfológicos las parcelas (pendiente, cubierta vegetal, área y tipo suelo desnudo). El mejor resultado en este caso ha correspondido al total de escorrentía y de sedimentos con el suelo desnudo tipo 2 de manera negativa $(r=-0.46$ y $r=-0.41$. respectivamente, $\mathrm{p}<0.050)$. Debemos observar la disposición de los diversos ambientes en el espacio en las parcelas. Así, el comportamiento hidrológico de una parcela varía si la vegetación se distribuye a lo largo de la misma homogéneamente, a si se concentra en su zona alta o baja, como sucede en la 1.5b. La distancia entre la vegetación juega un papel fundamental porque puede controlar el volumen total de escorrentía generada en el área de

FIGURA 8

\section{COMPARACIÓN ENTRE LA ESCORRENTÍA OBSERVADA Y ESTIMADA EN LAS PARCELAS DE 4 Y 8 M SEGÚN LA ESCORRENTÍA EMITIDA POR LAS PARCELAS DE 1.5 M.}

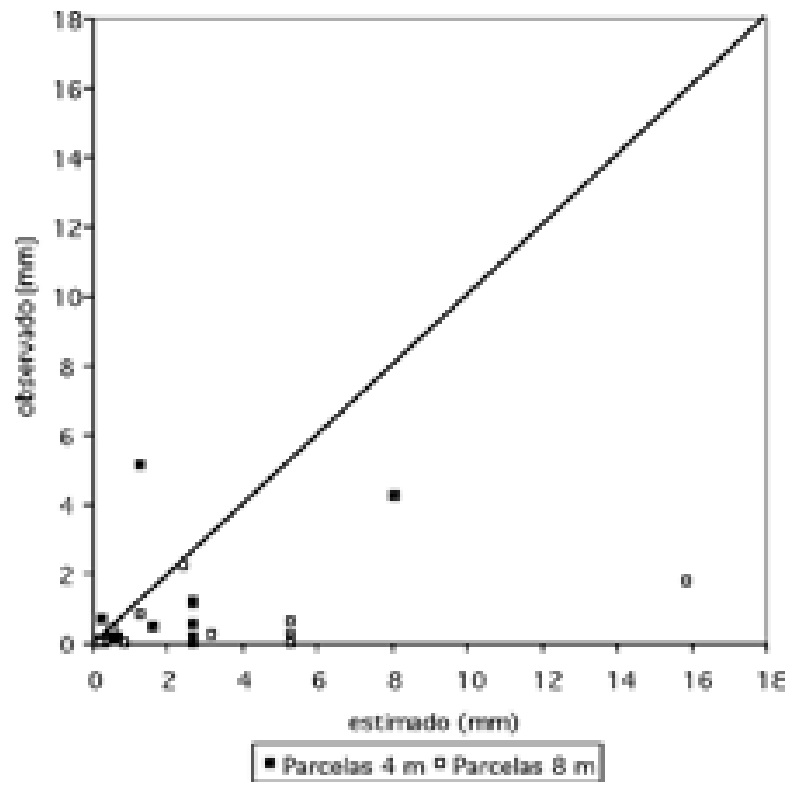


suelo desnudo como se ha señalado en varias investigaciones (Puigdefábregas y Sánchez 1996b; Bergkamp et al. 1999; Puigdefábregas et al. 1999). No ha habido buena relación entre estas variables y el volumen de la precipitación total y los sedimentos, lo que si fue obtenido por Bracken y Kirkby (2005) en laderas bajo régimen semiárido.

A pesar de la no abundancia de eventos lluviosos relevantes podemos apuntar que la evolución espacial y temporal del flujo superficial ladera abajo es bastante compleja, debido a la sucesión e interacción de las distintas unidades y condiciones eco-geomorfológicas. Ello nos permite intuir una cierta desconexión hidrológica entre los distintos ambientes dados sus diferentes comportamientos hidrológicos.

\section{DISCUSIÓN}

En las unidades analizadas hemos constatado un diverso comportamiento hidrodinámico como consecuencia del contenido de humedad del suelo, que ha actuado como principal factor regulador de su funcionamiento hidrológico y erosivo. Los experimentos al principio de la estación hidrodinámica activa han generado escorrentía con más frecuencia. La unidad SHV ha sido la que ha registrado más flujo superficial, siendo la que ha tenido más dificultades para infiltrar el agua pues solo un experimento ha superado un contenido de humedad del $20 \%$, después de 30 minutos de precipitación a $36.0 \mathrm{~mm} \mathrm{~h}^{-1}$. Las condiciones de gran sequedad del suelo observadas en el campo y los resultados de los experimentos nos hace apuntar que esa generación de escorrentía puede responder a la saturación de los primeros centímetros de suelo (SHV es la unidad con la conductividad hidráulica más baja como consecuencia de una peor estructura dado su menor contenido de materia orgánica). Puede apuntarse a la hidrofobia como la causa de control de los procesos de generación de escorrentía como otros autores han señalado (De Bano 1971; Witter et al. 1991; Ritsema y Dekker 1994; Doerr y Thomas 2000; Doerr et al. 2000; Ferreira et al. 2000) porque no hemos observado encostramientos en la superficie del suelo que pudieran impedir la infiltración del agua en el mismo. La escorrentía hortoniana domina en este momento de la estación hidrodinámica activa. Ferreira et al. (2000) observaron lo mismo durante el verano en suelos muy secos. También hemos registrado escorrentía bajo matorral de alta y baja densidad. El suelo de estas unidades ha mostrado un comportamiento hidrofóbico qué puede estar en relación con la textura más arcillosa bajo el matorral de la ladera. Sin embargo, la infiltración ha predominado en estas unidades lo qué ha conducido con frecuencia a que se supere un contenido de humedad del suelo del $20 \%$ a 5 centímetros. A 10 centímetros, el contenido de humedad 
no ha obtenido altos valores de modo que se ha observado también hidrofobia en el suelo. Estos valores tan bajos se relacionan igualmente con la elevada densidad de la cubierta vegetal, como ocurre en la Genista umbellata bajo la cuál la densidad foliar y su estructura pueden retrasar la llegada del agua a la superficie del suelo.

La emisión de sedimentos ha sido muy peculiar en algunos puntos pues inicialmente se incrementaba con la cubierta y la densidad foliar, lo que tiene que ser relacionado con el estado hídrico del suelo previo al evento simulado, aunque evidentemente se trataba sólo de una situación transitoria. El transporte de sedimentos ha sido mayor en los experimentos de precipitación de las unidades de HDS y de LDS que la unidad SHV. Esto puede poner de relieve dos hechos: i) las zonas de suelo desnudo están muy lixiviadas o lavadas superficialmente al menos, a causa de una escorrentía más frecuente, y ii) las zonas de matorral presentan una reserva muy importante de sedimento, bien generado in situ o bien procedente ladera arriba, y que puede ser movilizado por el flujo superficial. La estructura vegetal de cierta especie puede explicarlo: Genista Umbellata tiene una estructura aérea que tiende a concentrar flujo cortical en un punto sobre la superficie del suelo, la base de su tallo. Resultados análogos sobre las relaciones entre los sedimentos, la vegetación y el suelo desnudo han sido observados por Puigdefábregas y Sánchez (1996a), Puigdefábregas et al. (1998 y 1999).

El funcionamiento del sistema es más uniforme a mediados de la estación hidrodinámica activa. El suelo ha perdido su comportamiento hidrofóbico tras diferentes eventos pluviométricos a lo largo de la estación otoñal. La consecuencia es la superación de un umbral crítico de humedad del suelo que se puede definir entre unas condiciones hidrofóbicas y otras hidrofílicas (Soto et al. 1994). De hecho, la hidrofobia del suelo es una característica dinámica que presenta cambios a corto plazo y estacionales (Doerr y Thomas, 2000; Ferreira et al. 2000; McHale et al. en prensa) pasando a tener unas condiciones favorables para la infiltración del agua. Esta nueva situación se demuestra por la ausencia de escorrentía superficial en las unidades de matorral y suelo con vegetación herbácea fresca, esta última tan sólo emite flujo superficial cuando la pendiente aumenta por encima del $20 \%$. Los mecanismos de generación de escorrentía se han modificado y la saturación del suelo predomina como principal mecanismo a mediados de la estación hidrodinámica debido a una mayor capacidad de infiltración (Ferreira et al. 2000). Por lo tanto el transporte de sedimento queda muy limitado a zonas concretas con escasa cubierta vegetal y elevada pendiente. A mediados de la estación hidrodinámica activa, los sedimentos transportados son menos abundantes que al principio, lo que debe estar relacionado con el crecimiento de la vegetación herbácea anual que retiene los sedimentos, y la menor capacidad de arrastre de la escorrentía. 
Podemos observar en la tabla 4 la importancia de la humedad del suelo y la evolución desde unas condiciones hidrofóbicas a otras hidrofílicas. Los cambios son significativos en unidades de matorral en cuanto a escorrentía y al transporte de sedimento pero sobre todo en la unidad SHV. La estación seca sin eventos de precipitación provoca una extrema ausencia de agua en el suelo el cual llega a estar muy por debajo del punto de marchitez. Esta extrema sequedad va a originar una respuesta hidrófila del suelo ante las primeras lluvias otoñales. La consecuencia es una generalizada situación de generación de escorrentía superficial al inicio de la estación hidrodinámica activa. La diferente humedad del suelo produce una diferenciación entre las primeras y segundas partes de las simulaciones de la precipitación: la humedad más alta en los primeros centímetros del suelo causa el aumento de la escorrentía. Al contrario, el factor de la humedad del suelo presenta en principio menos importancia a mediados de la estación hidrodinámica, una vez que se supera la hidrofobia, lo cual se manifiesta en el comportamiento infiltrador del matorral. La escorrentía y la emisión de sedimentos se reducen a valores mínimos.

En estos procesos hidrológicos descritos, la distribución de los contenidos de arcilla tiene una incidencia directa. Superficialmente, el suelo bajo matorral presentaba una textura franco-arcillosa, con contenidos de arcilla superiores al $25 \%$. Ante unas condiciones de extrema sequedad y sin agua útil en el suelo, el suelo puede sufrir procesos de retracción o pérdida de volumen reduciéndose al mismo tiempo la porosidad (Kutilek y Nielsen 1994). La consecuencia es la reducción de la capacidad de infiltración del suelo favoreciendo los fenómenos hidrófobos señalados y que se traducían en emisión de escorrentía incluso en áreas de matorral. En las áreas de suelo desnudo, con unos primeros centímetros muy lavados y elevada pedregosidad superficial, las condiciones para la infiltración parecían más adecuadas. Pero apenas unos $5 \mathrm{~cm}$ bajo superficie, el contenido de arcillas es similar que en el suelo bajo matorral, de forma que la escorrentía está favorecida por unas condiciones edáficas e hidrofóbicas similares en las áreas de suelo desnudo, que emiten una vez saturado. Ello podía ser observado en el tiempo de concentración de la escorrentía más elevado. 
TABLA 4

COMPARACIÓN DE LAS RESPUESTAS HIDROLÓGICAS DE LAS MICRO-PARCELAS DE SIMULACIÓN SEGÚN LA FASE TEMPORAL DE LA ESTACIÓN HIDRODINÁMICA Y EL CONTENIDO DE AGUA EN EL SUELO

\begin{tabular}{|c|c|c|c|c|c|}
\hline Fase & Fecha & Humedad del suelo & HDS & LDS & SHV \\
\hline $1^{\mathrm{a}}$ & Oct-04 & Muy baja $(\mathrm{H}<5.0 \%)$ & $1 / 5$ & $2 / 5$ & $3 / 5$ \\
\hline $2^{\mathrm{a}}$ & Oct-04 & Baja $(\mathrm{H}>5.0 \%)$ & $2 / 5$ & $2 / 5$ & $3 / 5$ \\
\hline $1^{\mathrm{a}}$ & Mar-05 & Media $(\mathrm{H}>8.0 \%)$ & $0 / 5$ & $0 / 5$ & $2 / 5$ \\
\hline $2^{\mathrm{a}}$ & Mar-05 & Alta $(\mathrm{H}>15.0 \%)$ & $0 / 5$ & $0 / 5$ & $2 / 5$ \\
\hline
\end{tabular}

Fase: $1^{\mathrm{a}}$ y $2^{\mathrm{a}}$ partes de los experimentos de simulaciones de lluvia.

Estas últimas afirmaciones se corresponden con lo que ocurre en una micro-parcela en el momento exacto en el que se inicia la escorrentía y, desde ahí, la escorrentía debe aumentar en volumen al incrementarse la distancia recorrida ladera abajo siguiendo un esquema hortoniano (Kirkby y Chorley 1967; Hagget y Chorley 1969; Kirkby 1969; Carson y Kirkby 1972). Sin embargo, esta dinámica no ha sucedido en las parcelas cerradas, de lo que se desprende la falta de conectividad hidrológica entre los elementos del sistema biótico. De hecho, a lo largo de la ladera el patrón de la vegetación es discontinuo definiendo áreas o bandas de vegetación perpendiculares a la pendiente, delimitadas por áreas de suelo desnudo, y por tanto sucediéndose áreas potenciales infiltradoras y generadoras.

Por el contrario, el total de sedimentos emitidos ha crecido con la longitud de dichas parcelas. Bracken y Kirkby (2005) obtuvieron un resultado similar respecto a la movilización de la cantidad parcial y total de sedimentos en laderas metamórficas. La evolución temporal de la escorrentía y el transporte de sedimentos que se registró en las parcelas, puede ser correlacionada con las simulaciones de lluvia. Al principio de la estación hidrodinámica activa, la escorrentía y los sedimentos son abundantes y la infiltración está tan limitada que el contenido de humedad del suelo es muy bajo presentando síntomas de hidrofobia. Pero una vez que cambia el estado hídrico del suelo, la vegetación herbácea anual crece, aumenta la capacidad de infiltración del suelo y se reduce la escorrentía superficial y el transporte de sedimentos. 


\section{CONCLUSIONES}

Analizados los diferentes comportamientos hidrodinámicos en la ladera, podemos concluir que:

Después de un verano largo y seco como es característico de condiciones mediterráneas, el contenido en agua del suelo es muy bajo (por debajo del punto de marchitez) y el suelo presenta un comportamiento hidrofóbico. La consecuencia es la reducción de la capacidad de infiltración del suelo a pesar de que las condiciones superficiales del suelo parecen favorecer la infiltración del agua (elevado contenido de fragmentos rocosos no embebidos y sin encostramientos sobre el suelo). Así pues, los mecanismos hortonianos dominan los procesos de generación de escorrentía durante la primera parte de la estación hidrodinámica activa (septiembre a diciembre).

Una vez que el suelo se ha humedecido, incrementa su capacidad de infiltración, modificándose los mecanismos de generación de escorrentía. Esta tiende hacia la saturación del suelo lo que puede observarse en la ausencia de escorrentía en las simulaciones de lluvia, así como en las parcelas cerradas.

El transporte de sedimentos sucede principalmente durante los primeros eventos de precipitación cuando el suelo está más desprotegido. Los sedimentos cubren una distancia corta en cada evento moviéndose ladera abajo y tan sólo precipitaciones intensas parecen poder provocar un desplazamiento mayor. De este modo, se establece un ciclo en el transporte de sedimentos ladera abajo, relacionado con el mecanismo de generación de escorrentía y la intensidad de la precipitación lo cual ha de tener una trascendencia muy importante en el suministro de material fino para el suelo existente bajo la vegetación de matorral.

Aunque la información obtenida de las parcelas cerradas no ha sido especialmente abundante, hemos podido observar cierta variabilidad respecto a la generación de escorrentía superficial y el transporte de sedimentos a lo largo de distintos segmentos de longitud de la ladera analizada. Es evidente la alta variabilidad de la generación de escorrentía en laderas mediterráneas poniendo de manifiesto la importancia de la conectividad hidrológica, puesto que en su movimiento, el flujo superficial sufre modificaciones espaciales y temporales. 


\section{BIBLIOGRAFÍA}

BERGKAMP, G. (1998a): Hydrological influences on the resilience of Quercus spp dominated geoecosystems in central Spain. Geomorphology, 23, 101-126.

BERGKAMP, G. (1998b): A hierarchical view of interactions of runoff and infiltration with vegetation and microtopography in semiarid shrublands. Catena 33, 201-220.

BERGKAMP, G., CERDÁ, A., IMESON, A.C. (1999): Magnitude-frequency analysis of water redistribution along a climate gradient in Spain. Catena, 37, 129146.

BEVEN, K. (2002): Runoff generation in semi-arid areas, en BULL, L.J., M.J. KIRKBY (Eds), Dryland rivers, Hydrology and Geomorphology of Semi-Arid Zones. Wiley, 57-105.

BOIX, C. (1999): Procesos geomórficos en diferentes condiciones ambientales mediterráneas: el estudio de la agregación y la hidrología de suelos. Tesis doctoral, Universidad de Valencia..

BRACKEN, L.J., KIRKBY, M.J. (2005): Differences in hillslope runoff and sediment transport rates within two semi-arid catchments in southeast Spain. Geomorphology, 68, 183-200.

CALVO, A., GISBERT, J., PALAU, E., ROMERO, M. (1988): Un simulador de lluvia portátil de fácil construcción, en M. SALA y F. GALLART (Eds): Métodos y técnicas para la medición de procesos geomorfológicos. Monografías de la SEG 1, 6-15.

CALVO, A., BOIX, C., IMESON, A.C. (2003a): Runoff generation, sediment movement and soil water behavior on calcareous (limestone) slopes of some Mediterranean environments in Southeast Spain. Geomorphology, 50, 269-291.

CALVO, A., RUIZ, J.D., SARAH, P, KATRA, I., ZVIKEL, S., ARNAU, E., LAVEE, H. (2003b): The spatial distribution of surface cover components that control geomorphic processes on hillslopes along a Mediterranean-Arid transect.

CAMMERAAT, L.H. (2002): A review of two strongly contrasting geomorphological systems within the context of scale. Earth Surface Processes and Landforms, 27, 1201-1222.

CANTÓN, Y., SOLÉ-BENET, A., DOMINGO, F. (2004): Temporal and spatial patterns of soil moisture in semiarid badlands of SE Spain. Journal of Hydrology 285, 199-214.

CARSON, M.A., KIRKBY, M.J. (1972): Hillslope Form and Process. Cambridge University Press.

CASTILLO, V.M., GÓMEZ-PLAZA, A., MARTÍNEZ-MENA, M. (2003): The role of antecedent soil water content in the runoff response of semiarid catchments: a simulation approach. Journal of Hydrology, 284, 114-130.

CEBALlOS, A., MARTÍNEZ-FERNÁNDEZ, J., SANTOS, F., ALONSO, P. (2002): Soil-water behaviour of sandy soils under semiarid conditions in the Duero Basin (Spain). Journal of Arid Environments, 51, 501-519.

CERDÂ i BOLINCHES, A. (1995): Factores y variaciones espacio-temporales de la infiltración en los ecosistemas mediterráneos. Geoforma, Logroño, Spain. 
CERDÀ, A. (1997): The effect of patchy distribution of Stipa tenacissima L. on runoff and erosion. Journal of Arid Environments, 36, 37-51.

CERDÀ, A. (1998a): Seasonal changes of the infiltration rates in a Mediterranean scrubland on limestone. Journal of Hydrology, 198, 209-225.

CERDÀ, A. (1998b): The influence of geomorphological position and vegetation cover on the erosional and hydrological processes on a Mediterranean hillslope. Hydrological Processes, 12, 661-671.

CERDÀ, A., IMESON, A.C., CALVO, A. (1995): Fire and aspects induced differences on the erodibility and hydrology of soils at La Costera, Valencia, SE Spain. Catena, 24, 289-304.

DE BANO, L.F. (1971): The effect of hydrophobic substances on water movement in soil during infiltration. Soil Science Society of America Proceedings, 35, 340343.

DEKKER, L.W., RITSEMA, C.J. (1994): How water moves in a water repellent sandy soil 1. Potential and actual water repellency. Water Resources Research, 30, 2507-2517.

DOERR, S.H., THOMAS, A.D. (2000): The role of soil moisture in controlling water repellency: new evidence from forest soils in Portugal. Journal of Hydrology, 213-232, 134-147.

DOERR, S.H., SHAKESBY, R.A., WALSH, R.P.D. (2000): Soil water repellency: its causes, characteristics and hydro-geomorphological significance. Earth Science Reviews 51, 33-65.

FERREIRA, A.J.D., COELHO, C.O.A., WALSH, R.P.D., SHAKESBY, R.A., CEBALLOS, A., DOERR, S.H. (2000): Hydrological implications of soil waterrepellency in Eucalyptus globulus, north-central Portugal. Journal of Hydrology, 231-232, 165-177.

GÜNTNER, A., BRONSTERT, A. (2004): Representation of landscape variability and lateral redistribution processes for large-scale hydrological modelling in semi-arid areas. Journal of Hydrology, 297, 136-161.

HAGGET, P., CHORLY, R.J. (1969): Network Analysis in Geography. Arnold, London.

IMESON, A.C., VERSTRATEN, J.M., VAN MULLIGEN, E.J., SEVINK, J. (1992): The effects of fire and water repellency on infiltration and runoff under Mediterranean type forests. Catena, 19, 345-361.

INGELMO, F., CUADRADO, S., IBÁÑEZ, A., HERNÁNDEZ, J. (1994): Hydric properties of some Spanish soils in relation to their fragment content: implication for runoff and vegetation. Catena, 23, 73-85.

JUSTICIA-SEGOVIA, A. (1988): La Axarquía malagueña y la Costa oriental. Dos espacios agrarios contrapuestos. Arguval, Málaga, Spain.

KIRKBY, M.J. (1969): Infiltration, throughflow and overland flow, en CHORLEY, R.J. (Ed.): Water, Earth and Man, Methuen, London, 215-227.

KIRKBY, M.J., CHORLEY, R.J. (1967): Throughflow, overland flow and erosion. Bull. Inntern. Assoc. Sci. Hydrology, 12, 5-21.

KUTILEK, M., NIELSEN, D.R. (1994): Soil Hydrology. GeoEcology, Catena Verlag. 
LAVABRE, J., TORRES, D.S., CERNESSON, F. (1993): Changes in the hydrological response of a small Mediterranean basin a year after a wildfire. Journal of Hydrology, 142, 273-299.

LAVEE, H., IMESON, A.C., SARAH P. (1998): The impact of climate change on geomorphology and desertification along a mediterranean arid transect. Land Degradation and Development, 9, 407-422.

MARTÍNEZ-FERNÁNDEZ, J. (1996): Variabilidad espacial de las propiedades físicas e hídricas de los suelos en medio semiárido mediterráneo. Tesis doctoral, Universidad de Murcia.

MARTÍNEZ-MURILLO, J.F. (2006): Variabilidad espacio-temporal del sistema ecogeomorfológico mediterráneo en un ambiente ecotono semiárido/subhúmedo de los Montes de Málaga. Implicaciones hidrológicas y erosivas. Tesis doctoral, Universidad de Málaga.

McHALE, G., NEWTON, M.I., SHIRTCLIFFE, N.J. (en prensa): Water-repellent soil and its relationship to granularity surface roughness and hydrophobicity: a material science review. European Journal of Soil Science.

NICOLAU, J.M., SOLÉ, A., PUIGDEFÁBRGAS, J., GUTIÉRREZ, L. (1996): Effects of soil and vegetation on runoff along a catena in semi-arid Spain. Geomorfphology, 14, 297-309.

PEREZ-LATORRE, A., NIETO-CALDERA, J.M., CABEZUDO, B. (1994): Datos sobre la vegetación de Andalucía III. Series de vegetación caracterizada por Quercus suber L. Acta Botánica Malacitana 19, 169-183.

PUIGDEFÁBREGAS, J. (1998): Ecological impacts of global change on drylands and their implications for desertification. Land degradation and Management, 9, 393-406.

PUIGDEFÁBREGAS, J., SÁNCHEZ, C. (1996a): Vegetación dispersa y flujo de vertiente en medio semiárido, en GRANDAL, A., PAGÉS, J. (Eds): Actas de la IV Reunión de Geomorfología de la Sociedad Española de Geomorfología, Cadernos de Laboratorio Xeoloxio de Laxe 21, 375-392.

PUIGDEFÁBREGAS, J., SÁNCHEZ, G. (1996b): Geomorphological implications of vegetation patchiness on semi-arid slopes, en ANDERSON, M.G., BROOKS, S. (Eds): Advances in Hillslope Processes 2. Wiley, 1027-1060.

PUIGDEFÁBREGAS, J., DEL BARRIO, G., BOER, M.M., GUTIÉRREZ, L., SOLÉ, A. (1998): Differential responses of hillslope and channel elements to rainfall events in a semiarid area. Geomorphology, 23, 337-351.

PUIGDEFÁBREGAS, J., SOLÉ, A., GUTIÉRREZ, L., DEL BARRIO, G., BOER, M. (1999): Scales and processes of water and sediment redistribution in drylands: results from the Rambla Honda field site in Southeast Spain. Earth-Science Reviews, 48, 39-70.

RAMOS, M.C., MULLIGAN, M. (2005): Spatial modelling of the impact of climate variability on the annual soil moisture regime in mechanized Mediterranean vineyard. Journal of Hydrology, 36, 287-301.

RITSEMA, C.J., DEKKER, L.W., HENDRICKX, J.M.H., HAMMINGA, W. (1993): Preferential flow mechanism in a water repellent sandy soil. Water Resources Research, 29, 2183-2193. 
RITSEMA, C.J., DEKKER, L.W. (1994): How water moves in a water repellent sandy soil. 2. Dynamics of fingered flow. Water Resources Research, 30, 2519-2531.

RÖMKENS, M.J.M., HLEMING, K., PRASAD, S.N. (2001): Soil erosion under different rainfall intensities, surface roughness and soil water regimes. Catena, 46, 103-123.

RUIZ-SINOGA, J.D., DELGADO-PEÑA, J.J., GALLEGOS-REINA, A., LUCASSANTAMARÍA, B., MARTÍNEZ-MURILLO, J.F., MÁRQUEZ-CARRERO, J., ROMERO-LOPERA, A. (2004): Procesos hidrológicos en microambientes bajo condiciones mediterráneas. Baetica, 26, 129-153.

SCOTT, D.F. (1992): The influence of vegetation type on soil wettability. Proceedings of the 17th Congress of the Soil Science Society of South Africa, University of Stellenbosch, 28-30 th Jan. 1992. 10B21-10B26.

SCOTT, D.F., VAN WYK, D.B. (1990): The effects of wildfire on soil wettability and hydrological behaviour of an afforested catchment. Journal of Hydrology, $121,239-256$.

SCOTT, D.F., SCHULZE, R.E. (1992): The hydrological effects of a wildfire in a eucalypt afforested catchment. South African Forestry Journal, 160, 67-74.

SENCIALES-GONZÁLEZ, J.M. (1995): La cuenca del río Vélez, estudio hidrográfico. Tesis doctoral, Universidad de Málaga.

SHAKESBY, R.A., COELHO, C.O.A., FERREIRA, A.D., TERRY, J.P., WALSH, W.P.D. (1993): Wildfire impacts on soil erosion and hydrology in wet Mediterranean forest, Portugal. International Journal of Wildland Fire, 3, 95-110.

SOTO, B., LASANTA, R., BENITO, E., PEREZ, R., DIAZ-FIERROS, F. (1994): Runoff and erosion from burnt soils in northwest Spain, en SALA, M., RUBIO, J.L. (Eds): Soil erosion as a consequence of forest fires, Geoforma Ediciones, Logroño, 91-98.

WAINWRIGHT, J., PARSONS, A.J., SCHLESINGER, W.H., ABRAHAMS, A.D. (2002): Hydrology-vegetation interactions in areas of discontinuous flow on a semi-arid bajada, Southern New Mexico. Journal of Arid Environments, 51, 319338.

WITTER, J.V., JUNGERIUS, P.D., TEN HARKEL, M.J. (1991): Modelling water erosion and the impact of water repellency. Catena 18, 115-124.

YAIR, A., KLEIN, Y. (1973): The influence of surface properties on flow and erosion processes on debris covered slopes in an arid area. Catena, 1, 1-14.

YAIR, A., LAVEE, H. (1974): Areal contribution to runoff on scree slopes in an extreme arid environment. A simulated rainfall experiment. Zietschirft fur Geomorphologie N.F. Supplement 21, 106-121.

YAIR, A., LAVEE, H. (1985): Runoff generation in arid and semi-arid zones. Hydrological Forecasting, 133, 433-446.

YAIR, A., ENZEL, Y. (1987): The relationship between annual rainfall and sediment yield in arid and semiarid areas. The case of the northern Negev. Catena Supplement, 10, 121-135. 
